\title{
Locally Convex Words and Permutations
}

\author{
Christopher Coscia \\ Department of Mathematics \\ Dartmouth College \\ New Hampshire, U.S.A. \\ christopher.s.coscia.gr@dartmouth.edu \\ Jonathan DeWitt \\ Department of Mathematics and Statistics \\ Haverford College \\ Pennsylvania, U.S.A \\ jdewitt@haverford.edu
}

Submitted: Jul 7, 2015; Accepted: Mar 29, 2016; Published: Apr 15, 2016

Mathematics Subject Classifications: 05A05, 05A15, 05A16, 05A17

\begin{abstract}
We introduce some new classes of words and permutations characterized by the second difference condition $\pi(i-1)+\pi(i+1)-2 \pi(i) \leqslant k$, which we call the $k$ convexity condition. We demonstrate that for any sized alphabet and convexity parameter $k$, we may find a generating function which counts $k$-convex words of length $n$. We also determine a formula for the number of 0 -convex words on any fixed-size alphabet for sufficiently large $n$ by exhibiting a connection to integer partitions. For permutations, we give an explicit solution in the case $k=0$ and show that the number of 1-convex and 2-convex permutations of length $n$ are $\Theta\left(C_{1}^{n}\right)$ and $\Theta\left(C_{2}^{n}\right)$, respectively, and use the transfer matrix method to give tight bounds on the constants $C_{1}$ and $C_{2}$. We also providing generating functions similar to the continued fraction generating functions studied by Odlyzko and Wilf in the "coins in a fountain" problem.
\end{abstract}

\section{Introduction}

In this paper we investigate permutations $\pi \in S_{n}$ and words (functions $f:[n] \rightarrow[p]$ ) that obey the condition:

$$
\pi(i-1)+\pi(i+1)-2 \pi(i) \leqslant k \quad \text { for all } i \in[2, \ldots, n-1],
$$

and

$$
f(i-1)+f(i+1)-2 f(i) \leqslant k \quad \text { for all } i \in[2, \ldots, n-1],
$$

respectively, where $k \in \mathbb{Z}_{n \geqslant 0}$ and $[n]=\{1,2, \ldots, n\}$. We refer to these as locally convex permutations and words with respect to $k$, or $k$-convex permutations and words. Geometrically, 0-convex permutations and words, such as 1342 or 14444322, respectively, 
are those such that when the permutation or word entries are plotted against their positions and consecutive entries are connected by straight line segments, the area under the plot is convex. This description is different than that presented in [2], in which the authors consider the polygon enclosed by the plotted points. Intuitively, an increase in the parameter $k$ represents a relaxation of this condition.

The study of these permutations arises from a problem in a graduate course taught by Jamie Radcliffe and brought to our attention by Jessie Jamieson. The original problem is stated as follows:

Problem 1.1. Let $\sigma \in S_{n}$. If, for all $i \in[n-1]$, we have:

$$
\sigma(i+1) \leqslant \sigma(i)+1
$$

then $\sigma$ is a slow riser. Let $S l o w_{n}$ be the number of slow risers in $S_{n}$. What is $S l o w_{n}$ ?

Slow $w_{n}$ is $2^{n-1}$ since for any slow riser $\sigma$ of length $n-1$, there are exactly two places in the one line notation for $\sigma$ in which we can insert $n$ to form a slow riser of length $n$; $n$ may be inserted immediately after $n-1$ or at the beginning of the permutation. This "extension" map is 1 to 2 as removing $n$ from a valid permutation of length $n$ gives us a valid permutation of length $n-1$.

We may abstract the property given above by noticing that it could equivalently be stated that $\sigma \in S_{n}$ is a slow riser if the first differences of $\sigma$ are bounded above by one. We can generalize this problem by selecting some value other than one (call it $k$ ) to work with, but the previous argument still works as long as we pick a suitable extension procedure, so we consider instead second differences. We thank Bill Kay for suggesting this generalization.

The generalization is natural in the sense that it is closely related to previous problem and keeps some of the same structure. In particular, it is easily seen that slow rising permutations are exactly those permutation which avoid the patterns 312 and 213, which are also called reversed layered permutations. Moreover, 0 -,1- and 2-convex permutations are also single-peaked as they avoid the patterns 312 and 213, but have additional structure making them strictly smaller than this class of permutations. Further, this generalization is also different as the set of $k$-convex permutations does not form a class (it is not closed downward under the permutation containment order).

First, we discuss locally convex words, which, in the case $k=0$, we enumerate by exploiting a bijection with pairs of integer partitions. In addition, we demonstrate that it is possible to derive a generating function for locally convex words for any $k$. We then study $k$-convex permutations, enumerating the set for $k=0$ and giving generating functions for the cases $k=1,2$. We show that in the cases $k=1,2$, the set of $k$-convex permutations grows exponentially, and we provide a method for extracting arbitrarily close estimates for the exponential bases by considering the graphs formed by tracking the growth of the sets by length.

The aforementioned generating functions which enumerate 1- and 2-convex permutations are quite similar to the generating function that describes the number of solutions to the "coins in a fountain" problem, as described in [10]. As such, further simplifications to our generating functions will be closely related to the study of the generating function for fountains, which admits a similar continued fraction, but does not seem to have a more tractable form. 


\section{Locally Convex Words}

We wish to count functions $f:[n] \rightarrow[p]$ such that for $i \in\{2, \ldots, n-1\}$ we have

$$
f(i-1)+f(i+1)-2 f(i) \leqslant k
$$

for some $k \in \mathbb{Z}_{\geqslant 0}$. We say that such a function is locally convex with parameter $k$. Notice that convex is an appropriate word to use here, as this stipulates a bound on the growth of the first differences:

$$
f(i+1)-f(i) \leqslant f(i)-f(i-1)+k \Longrightarrow f(i-1)+f(i+1)-2 f(i) \leqslant k
$$

Fix $p$ and $k$. Let $f_{n}(a, b)$, for $a, b \in[p]$, be the number of functions $f:[n] \rightarrow[p]$ such that $f(1)=a$ and $f(2)=b$ that also obey the convexity condition (2.1). We will determine the generating function:

$$
F(a, b)=\sum_{n \geqslant 2} f_{n}(a, b) x^{n}
$$

and when all of these generating functions have been determined, we will simply sum them in order to find the generating function for all such functions. That is,

$$
G_{k, p}=1+p x+\sum_{a, b \in[p]} F(a, b)
$$

We will now give a method of determining $F(a, b)$ given some fixed $p$ and $k$ using the Transfer Matrix method, which is described in [11], Section 4.7. For a description and examples of the transfer matrix method as used for enumerating permutations, see [7], [8], and [12].

These functions are in bijection with sequences $a_{1}, \ldots, a_{n}$ where $a_{i}=f(i)$, which we will discuss below. To begin, we claim that for $n \geqslant 3$, we have:

$$
f_{n}(a, b)=\sum_{i \leqslant k+2 b-a} f_{n-1}(b, i)
$$

Given $a_{1}, \ldots, a_{n}$, we know that $a_{2}, \ldots, a_{n}$ is unique and counted by $f_{n-1}\left(a_{2}, a_{3}\right)$. If $a_{1}, \ldots, a_{n-1}$ is a sequence such that $a_{1}=b$, then we see that this sequence has a unique extension to a sequence beginning with $a$ if and only if $a+a_{2}-2 a_{1} \leqslant k$, or equivalently, $a_{2} \leqslant k+2 a_{1}-a$. Then, summing over all possible $a_{2}$, we find the relation above.

We now claim that

$$
F(a, b)=x\left(\sum_{i \leqslant k+2 b-a} F(b, i)\right)+x^{2} .
$$

To see this, we multiply by $x^{n}$ and sum over (2.2) for $n \geqslant 3$. We have

$$
\sum_{n \geqslant 3} f_{n}(a, b) x^{n}=\sum_{n \geqslant 3} \sum_{i \leqslant k+2 b-a} f_{n-1}(b, i) x^{n} .
$$


Reversing the order of summation on the right and changing the indexing, we find:

$$
\sum_{n \geqslant 3} f_{n}(a, b) x^{n}=\sum_{i \leqslant k+2 b-a} x \sum_{n \geqslant 2} f_{n}(b, i) x^{n} .
$$

Now note that $\left[x^{2}\right] F(a, b)=1$ so we can add $x^{2}$ to both sides and conclude (2.3). Furthermore, note that we have $p^{2}$ such equation in $p^{2}$ unknowns, $F(a, b)$. To show that it is possible to find a generating function, it will suffice to check that such equations have a unique solution.

Note that we can rearrange the above equation as follows:

$$
x^{2}=F(a, b)-x\left(\sum_{i \leqslant k+2 b-a} F(b, i)\right) .
$$

Then we see that as the functions $F(a, b)$ satisfy a system of linear equations in $\mathbb{R}[[x]]$, finding these functions is a matter of linear algebra in $\mathbb{R}[[x]]$. In particular, if we let $A$ be the matrix associated to this system of equations, so that the vector of interest is $\vec{w}$ where $A \vec{w}=\vec{v}$ where $v=\left[x^{2}, \ldots, x^{2}\right]^{T} \in \mathbb{R}[[x]]^{p^{2}}$. It then suffices to check that $\operatorname{det} A$ is a unit in $\mathbb{R}[[x]]$ as this guarantees a unique solution; this is the case, as in [11]. Solving the linear equations for each $F(a, b)$ we are able to determine $G_{k, p}$.

As an example we can calculate that

$$
\begin{aligned}
G_{0,3} & =1+3 x+9 x^{2}+16 x^{3}+20 x^{4}+21 x^{5} /(1-x) \\
& =1+3 x+9 x^{2}+16 x^{3}+20 x^{4}+21 x^{5}+21 x^{6}+21 x^{7}+\ldots
\end{aligned}
$$

Notice that the number of such permutations as $n$ becomes large stays constant at the value of 21. Perhaps somewhat surprisingly, this is true for all values of $p$ when $k=0$; this is our next result.

Theorem 2.1. Let $g_{0, p}(n)=\left[x^{n}\right] G_{0, p}=\#\{f:[n] \rightarrow[p] \mid f(i-1)+f(i+1)-2 f(i) \leqslant$ $0 \forall i \in\{2,3, \ldots, n-1\}\}$, then for $n>2(p-1)$,

$$
g_{0, p}(n)=\sum_{m=1}^{p}\left(\sum_{j=0}^{m-1} a(j)\right)^{2}
$$

where $a(j)$ is the number of integer partitions of $j$.

Proof. We rewrite this sum as

$$
g_{0, p}(n)=\sum_{m=1}^{p} h(m)
$$

and see that $h(m)$ can be interpreted as the cardinality of $H_{m}$, which we define as the set of 0 -convex words of a fixed length at least $2 p-1$ on the alphabet $[p]$ that attain a maximal value of $m$. Now given a word $w \in H_{m}$, we will show that every element in $H_{m}$ is determined uniquely by an ordered pair $\left\{w_{1}, w_{2}\right\}$ of integer partitions of $m-w_{f}$ and $m-w_{\ell}$ for $w_{f}$ and $w_{\ell}$ the first and last entries of $w$, respectively, with possible values between 1 and $m$, which would mean that $h(m)=\left(\sum_{j=0}^{m-1} a(j)\right)^{2}$. 
Fix $m \leqslant p$ and let $w_{1}, w_{2}$ be two integer partitions of $m_{1}, m_{2}<m$, respectively. Write $w_{1}$ as a sequence with elements corresponding to the parts of the integer partitions written in increasing order. Now construct the prefix word $p f$, where for all $i=1,2, \ldots,\left|w_{1}\right|$,

$$
p f(i)=m-\left(\sum_{j=1}^{\left|w_{1}\right|-i+1} w_{1}(j)\right) .
$$

The result is a 0 -convex word of length at most $m_{1}$ whose first entry is $m-m_{1} \geqslant 1$; it is strongly increasing and 0-convex (the second difference condition is satisfied because the sequence of first differences is weakly decreasing while the entries themselves are increasing, by construction). Similarly write $w_{2}$, the sequence of partial sums of the partition of $m_{2}$, also in decreasing order, and form the word $s f$ where

$$
s f(i)=m-\left(\sum_{j=1}^{i} w_{2}(j)\right) .
$$

Then $s f$ is 0 -convex and strongly decreasing, with final element $m-m_{2}$. For $c \geqslant 0$, form a 0 -convex word of length $2 p-1+c$ that attains a maximum value of $m$ by appending $2 p-1+c-|p f|-|s f|$ copies of $m$ to $p f$ and then further appending $s f$ to the result; note that $2 p-1+c-|p f|-|s f| \geqslant 2 p-1+c-m_{1}-m_{2} \geqslant c+1$. To show that the concatenation of these three 0 -convex sequences is also 0 -convex, we must check the boundaries between the subwords. Clearly $2 m \geqslant m+p f\left(\left|w_{1}\right|\right)$ and $2 m \geqslant m+s f(1)$; notice also that by the construction of the words $p f$ and $s f$, the difference between the final two entries of $p f$ is less than the difference between $m$ and the final entry of $p f$, and because this sequence is increasing the second difference condition is satisfied. A similar argument holds for the transition between the $m$ plateau and $s f$, so we conclude that this construction maps the pair $\left\{w_{1}, w_{2}\right\}$ into $(p f) m \ldots m(s f)$, a 0 -convex word in $H_{m}$.

Next, fix $c \geqslant 0$ and let $w$ be a word of length $2 p-1+c$ that attains a maximum value of $m\left(w \in H_{m}\right)$. We know due to the convexity condition that $w$ may be written as some subset of a strictly increasing subword followed by a plateau consisting of the value $m$ and finally by a strictly decreasing subword; this ensures that all appearances of $m$ in $w$ are consecutive. Further, we know that $m$ appears at least once. We can then write $w=(p f) M(s f)$ where $p f$ and $s f$ are 0-convex, $p f$ is strongly increasing, $s f$ is strongly decreasing, and $M$ is a string of $m$ 's of length $2 p-1+c-|p f|-|s f| \geqslant$ $c+1>0$ (hence $w$ attains its maximum). Then $p f$ encodes a unique integer partition $w_{1}$ of $m-p f(1)$; the partition is given by the first differences of the entries in $p f$ and $m-p f\left(\left|w_{1}\right|\right)$ (the fact that this sequence is monotone decreasing proves uniqueness). As a similar argument can be made for $s f$ into the partition $w_{2}$ of $m-s f\left(\left|w_{2}\right|\right)$, we can now map the words $w=(p f) M(s f)$ into the pairs $\left\{w_{1}, w_{2}\right\}$ where $w \in H_{m}$. Thus, $h(m)=$ $\left|H_{m}\right|=\mid$ partitions of $0,1,2, \ldots, m-\left.1\right|^{2}=\left(\sum_{j=0}^{m-1} a(j)\right)^{2}$ and the result follows.

Example 1. In the following, we choose $n=2 p-1$, so $n$ is large enough that $g_{0, p}(\ell)$ is constant for $\ell \geqslant n$. Notice that the first entry of $p f$ encodes the difference between $p$ and the number partitioned by $w_{1}$, and the last entry of $s f$ encodes the difference between $p$ and the number partitioned by $w_{2}$. Let $p=8, w_{1}=\{1,1,2,3\}$ (a partition of 7 ), $w_{2}=\{2,4\}$ (a partition of 6 ), then $p f=1467, s f=62, w=146788888888862$.

The sequence $\left\{g_{0, p}(2 p-1)\right\}_{p}$ begins $1,5,21,70,214,575,1475,3500,7469, \ldots$ 


\section{Locally Convex Permutations}

Definition 3.1. Let $\pi$ be a permutation of length $n(\pi:[n] \rightarrow[n]$ is a bijection). We say that $\pi$ is $k$-convex for a nonnegative integer $k$ if it obeys the following:

$$
\pi(i-1)+\pi(i+1)-2 \pi(i) \leqslant k \text { for all } i \in\{2,3, \ldots, n-1\}
$$

If the statement is true for $k=0$, we call $\pi$ perfectly convex, and if the statement is true for $k=2$ (and, as a result, for $k=1$ and $k=0$ ), we say that $\pi$ is strongly convex.

Throughout, we will write $\pi$ in one line notation as $\pi(1) \pi(2) \pi(3) \ldots \pi(n)$. We are interested in enumerating the $k$-convex permutations of length $n$, which we define as $f_{k}(n)$. First we will establish a few elementary properties of permutation convexity. Techniques similar to ours for enumeration and estimating growth rates may be found in [1] and [4].

Proposition 3.2. If $\pi$ is a $k$-convex permutation of length $n$, then $\pi^{R}$, the reverse of $\pi$ in $S_{n}$, is also k-convex.

Proposition 3.3. Strongly convex permutations (and therefore perfectly convex permutations) avoid consecutive entries order-isomorphic to 213 or 312.

Proof. Suppose without loss of generality that, for some $i, \pi(i)<\pi(i-1)<\pi(i+1)$ (ie. there exists a consecutive 213), then $\pi(i-1)+\pi(i+1)-2 \pi(i)=(\pi(i+1)-\pi(i))-$ $(\pi(i)-\pi(i-1)) \leqslant 2+1=3$.

Notice that another way of phrasing this result is that strongly and perfectly convex permutations contain only substrings whose minima are located at either the beginning or the end of the substring. Further, we can say that 213 and 312 avoiding permutations consist of an increasing substring followed by a decreasing substring, making strongly convex permutations a "single-peaked". It can be easily seen that there are $2^{n-1}$ such permutations of length $n$. Thus we have our first upper bound on the number of strongly convex permutations:

Lemma 3.4. For $k \in\{1,2\}$,

$$
f_{k}(n)<2^{n-1} .
$$

(This is a strict inequality as clearly not all ascending-descending permutations are 2-convex.) There are other ways to obtain this result. One requires use of the fact that a strongly convex permutation of length $n$ inherits a strongly convex substring consisting of $[n-1]$. In other words, if a permutation of length $n-1$ is not strongly convex, there is no way to "fix" the permutation by inserting $n$. Let $\pi:[n-1] \rightarrow[n-1]$ be a permutation that is not strongly convex, so there is some $i$ for which $\pi(i-1)+\pi(i+1)-2 \pi(i)>2$. If one were to attempt to "fix" this permutation by extending $\pi$ to $\pi^{\prime}$, a permutation of length $n$, it is clear that one must insert $n$ next to $\pi(i)$ in the one line notation to fix the convexity condition. This will not work, however, as replacing $\pi(i-1)$ or $\pi(i+1)$ with $n$ will only worsen the convexity at this point.

Now, given a strongly convex permutation $\pi(1) \pi(2) \pi(3) \ldots \pi(n)$, how might one build a permutation of length $n+1$ ? The ascending-descending nature of the permutation 
dictates that if $\pi(i)=n$, it must be that either $\pi(i-1)=n-1$ or $\pi(i+1)=n-1$. For the same reason, it must be that the only possible positions in which we can place $n+1$ to extend $\pi$ to $\pi^{\prime}$ and preserve strong convexity are immediately before and immediately after $n$. Actually, one of these placements is always available; $\pi$ may always be extended to length $n+1$ by placing $n+1$ between $n$ and $n-1$. Doing this for each permutation, we attain the inequality

$$
1 \leqslant f_{k}(n+1) / f_{k}(n) \leqslant 2 \text { for } k=1,2 .
$$

This method of tracking growth preserves substrings by "building in the middle" of the permutation. A similar thing may be done by "building from the outside." We will discuss this method later.

We now give another result that will prove useful in enumerating strongly convex and perfectly convex permutations.

Proposition 3.5. If $\pi$ is $k$-convex with $k<3$, exactly one of the following must be true:

- $\pi(1)=1$ and $\pi(2)=2$, or

- $\pi(n)=1$ and $\pi(n-1)=2$, or

- $\pi(1)=1$ and $\pi(2)=3$, or

- $\pi(n)=1$ and $\pi(n-1)=3$, or

- $\pi(1)=2$ and $\pi(2)=3$, or

- $\pi(n)=2$ and $\pi(n-1)=3$.

Proof. Since these patterns are 213 and 312 avoiding, we see that either $\pi(1)=1$ or $\pi(n)=1$. We assume now that $\pi(1)=1$ and recall that since $\pi^{R}$ is also $k$-convex, it remains only to show that $\pi(2)=2$ or $\pi(2)=3$ or both $\pi(n)=2$ and $\pi(n-1)=3$. Suppose $\pi(2)>3$, then since $\pi$ is 213 and 312 avoiding (single-peaked, as described earlier), we know the decreasing sequence at the end of the permutation ends with the substring 32. This proves the proposition.

Using these facts, enumerating perfectly convex permutations is straightforward.

Theorem 3.6. For all $n \geqslant 1$,

$$
f_{0}(n)= \begin{cases}1 & \text { if } n=1 \\ 2 & \text { if } n=2 \\ 4 & \text { if } n=3 \\ 6 & \text { if } n=4 \\ 8 & \text { if } n \geqslant 5\end{cases}
$$

Proof. For $n=1,2$, this is trivially true, as the set $\{2, \ldots, n-1\}$ is empty. For $n=3$, it is easy to check that the only non-perfectly convex permutations are 213 and 312 , so $f_{0}(3)=3 !-2=4$. Now let $n \geqslant 4$; we will construct all perfectly convex permutations. 
To begin, assume that $\pi(1)=1$; this will count exactly half of the desired permutations. It is easy to check that the only permutations that satisfy the convexity condition are:

$$
\begin{gathered}
1234 \ldots n \\
1345 \ldots n 2 \\
1357 \ldots n \ldots 8642 \\
1 n \ldots 5432
\end{gathered}
$$

Notice that for $n=4$, permutations 3.2 and 3.3 are the same. The reverses of these permutations constitute the remaining perfectly convex permutations, hence the result.

Enumerating strongly convex permutations has proven much more difficult than the perfect case. We now give another method of tracking the growth of these permutations: by considering building "from the outside."

Definition 3.7. Let $\pi \in S_{n}$ be $k$-convex. Define

$$
L(\pi):=1(\pi(1)+1)(\pi(2)+1) \ldots(\pi(n)+1)
$$

and

$$
R(\pi):=(\pi(1)+1)(\pi(2)+1) \ldots(\pi(n)+1)(1)
$$

to be, respectively, the left and right descendants of $\pi$. If $L(\pi)$ is $k$-convex, we say that $\pi$ left descends, and similarly define what it means for a permutation to right descend.

Analogously, let $W=W_{1} W_{2} \ldots W_{n}$ be a word of length $n$ on the alphabet $\{L, R\}$, then $W(\pi)$ is the composition $W_{n}\left(W_{n-1}\left(\ldots\left(W_{2}\left(W_{1}(\pi)\right)\right) \ldots\right)\right)$. We say that $\tau$ is a descendant of $\pi$ and $\pi$ is an ancestor of $\tau$ if there exists some word $W$ on $\{L, R\}$ such that $W(\pi)=\tau$.

Proposition 3.8. Let $k<3$. If $\pi$ is a $k$-convex permutation of length $n$ with $\pi(1)=1$, then $L(\pi)$ is a $k$-convex permutation of length $n-1$. Similarly, if $\pi(n)=1$, then $R(\pi)$ is a $k$-convex permutation of length $n-1$.

Hence every $k$-convex permutation beginning with one descends from another $k$ convex permutation. For example, the 1-convex permutation 123564 descends from 12453 , and its reverse, 465321, descends from 35421, the reverse of the ancestor of the original permutation. This gives a mapping of strongly convex permutations of length $n$ (which must begin or end with 1) onto the strongly convex permutations of length $n-1$. Now consider this process in reverse; if $\pi$ is $k$-convex for $k \in\{0,1,2\}$ and $\pi(2)-2 \pi(1) \leqslant k$, then $L(\pi)$ is $k$-convex ( $\pi$ left descends), and similarly if $\pi(n-1)-2 \pi(n) \leqslant k$, then $R(\pi)$ is $k$-convex ( $\pi$ right descends). Notice that for $k=1$ or 2 , every $k$-convex permutation $\pi$ has either a $k$-convex right descendant or a $k$-convex left descendant, as we have shown previously that $\pi$ must begin with 12,13 , or 23 or end with 21,31 , or 32 , all of which satisfy the condition for a descendant to be $k$-convex.

Using this method to count and track strongly convex permutations, we determine $f_{k}(n)$ for $k \leqslant 2, n \leqslant 12$ (see Table 1 ). 


\begin{tabular}{cccc}
\hline$n$ & $f_{0}(n)$ & $f_{1}(n)$ & $f_{2}(n)$ \\
\hline 1 & 1 & 1 & 1 \\
2 & 2 & 2 & 2 \\
3 & 4 & 4 & 4 \\
4 & 6 & 8 & 8 \\
5 & 8 & 14 & 16 \\
6 & 8 & 24 & 30 \\
7 & 8 & 40 & 56 \\
8 & 8 & 66 & 102 \\
9 & 8 & 106 & 186 \\
10 & 8 & 170 & 336 \\
11 & 8 & 270 & 606 \\
12 & 8 & 426 & 1088
\end{tabular}

Table 1: Number of Strongly Convex Permutations for Small $n$

Definition 3.9. Let $\pi$ be a $k$-convex permutation of length $n$. Define

$$
\begin{aligned}
d_{k}(\pi, i): & =\mid k \text {-convex descendants of } \pi \text { of length } n+i \mid \\
& =\mid\left\{W \in\{L, R\}^{i} \mid W(\pi) \text { is } k \text {-convex }\right\} \mid
\end{aligned}
$$

We say that two permutations $\pi$ and $\tau$ are identically-descending with respect to $k$ if $d_{k}(\pi, i)=d_{k}(\tau, i)$ for all $i \in \mathbb{N}$. In this case, we write $d_{k}(\pi)=d_{k}(\tau)$.

Notice that by this definition, every permutation is identically-descending to its reverse, as the reverse permutation of $W(\pi)$ is $W^{C}\left(\pi^{R}\right)$, where $W^{C}$ is formed from $W$ by changing all $L$ 's to $R$ 's and all $R$ 's to $L$ 's. We generalize this in the following Lemma.

Lemma 3.10. Let $\pi$ be a permutation of length $n$ and let $\tau$ be a permutation of length $m$. If $\pi(1)=\tau(1), \pi(2)=\tau(2), \pi(n-1)=\tau(m-1)$, and $\pi(n)=\tau(m)$, or, if $\pi(1)=$ $\tau(m), \pi(2)=\tau(m-1), \pi(n-1)=\tau(2)$, and $\pi(n)=\tau(1)$, then $d_{k}(\pi)=d_{k}(\tau)$.

This is true simply because when considering whether the left and right descendants of a permutation are strongly convex, the only new condition to check is at the left or right endpoint, respectively. This suggests that when considering strongly convex permutations, we can abbreviate the permutations $1 a \ldots b c$ and $c b \ldots a 1$ by $1 a b c$ and not lose any information about its descendants. This result allows us to simplify the graph by removing some of the vertices, but it is possible to do even better:

Theorem 3.11. Fix $k \in\{0,1,2\}$ and let $\pi=a b \ldots c d$ and $\tau=a b \ldots c^{\prime} d$ be two $k$-convex permutations. If $R(\pi)$ and $R(\tau)$ are $k$-convex, then $d_{k}(\pi)=d_{k}(\tau)$.

We may now express many permutations by the same four-character abbreviation without losing any information about their descendants, choosing the smallest numbers possible for convenience. We previously displayed convex permutations as a directed tree with edges from permutations to their first descendants. We now display this graph allowing for cycles and reducing each permutation to the "smallest" abbreviation possible:

Let $A$ be the (infinite) adjacency matrix for the 1-convex descendant graph. We know then that since $A_{i j}^{n}$ is the number of walks of length $n$ from the $i^{\text {th }}$ vertex to the $j^{\text {th }}$ and 
The graph displayed here is half of the first seven generations of the full 1-convex tree showing descendants of 12 ; the other half begins with 21 and branches in the same manner except that each descendant is replaced by its reverse.
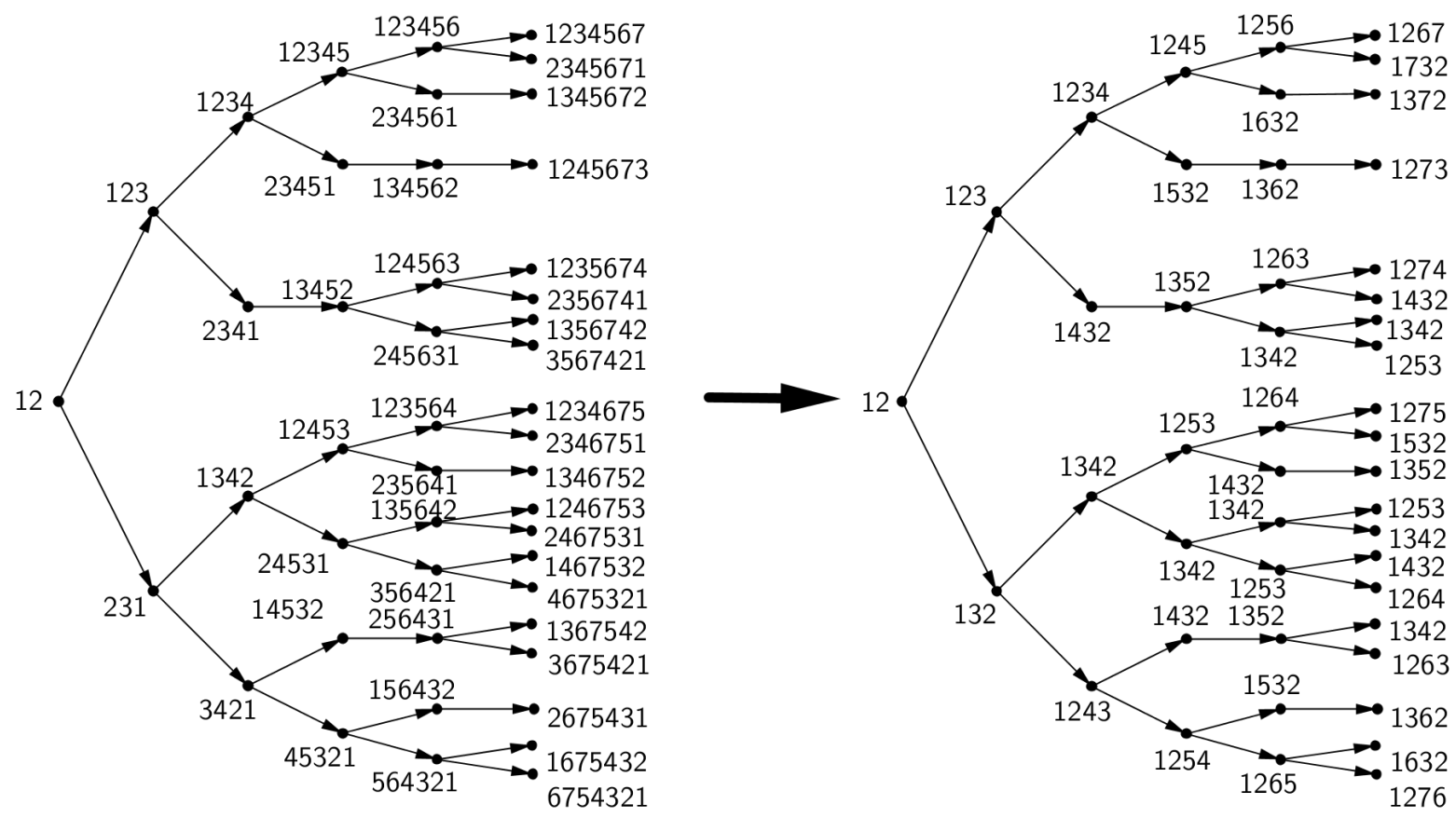

a 1-convex permutation of length $n+1$ is determined uniquely by a walk of length $n$ beginning at the vertex 12 , we have

$$
\sum_{j=1}^{\infty} A_{1 j}^{n-1}=\frac{1}{2} f_{1}(n) .
$$

We can obtain a lower bound on the growth rate of the sequence by truncating the graph at the edge from 1267 to 1278 (and therefore the corresponding adjacency matrix) and calculating $(I-A x)^{-1}$, which is formally equal to $\sum_{n=0}^{\infty} A^{n} x^{n}$. Each cell in the resulting matrix will contain a generating function for the corresponding cell of $A$. As we are interested in the number of walks beginning at the vertex 12, represented by the first row and column of the adjacency matrix, we sum over the first row to obtain a generating function for half the number of walks of length $n+1$. Using the first 22 rows and columns of the matrix (truncating the graph pictured above at 1267), we obtain:

$$
F_{1,-}(x)=\frac{-1-x-2 x^{2}-2 x^{3}-2 x^{4}-2 x^{5}-x^{6}+x^{7}+x^{8}+2 x^{9}+x^{10}+x^{11}-x^{14}}{-1+x+x^{3}+x^{4}-2 x^{8}-x^{9}-2 x^{10}+x^{13}+x^{15}}
$$

We now have a rational generating function whose denominator has a unique minimal root $r=0.65149869151455837735 \ldots$, which, as described in $[13$, p. 171] gives us an 
The same graph, identifying points abbreviated by the same four characters.

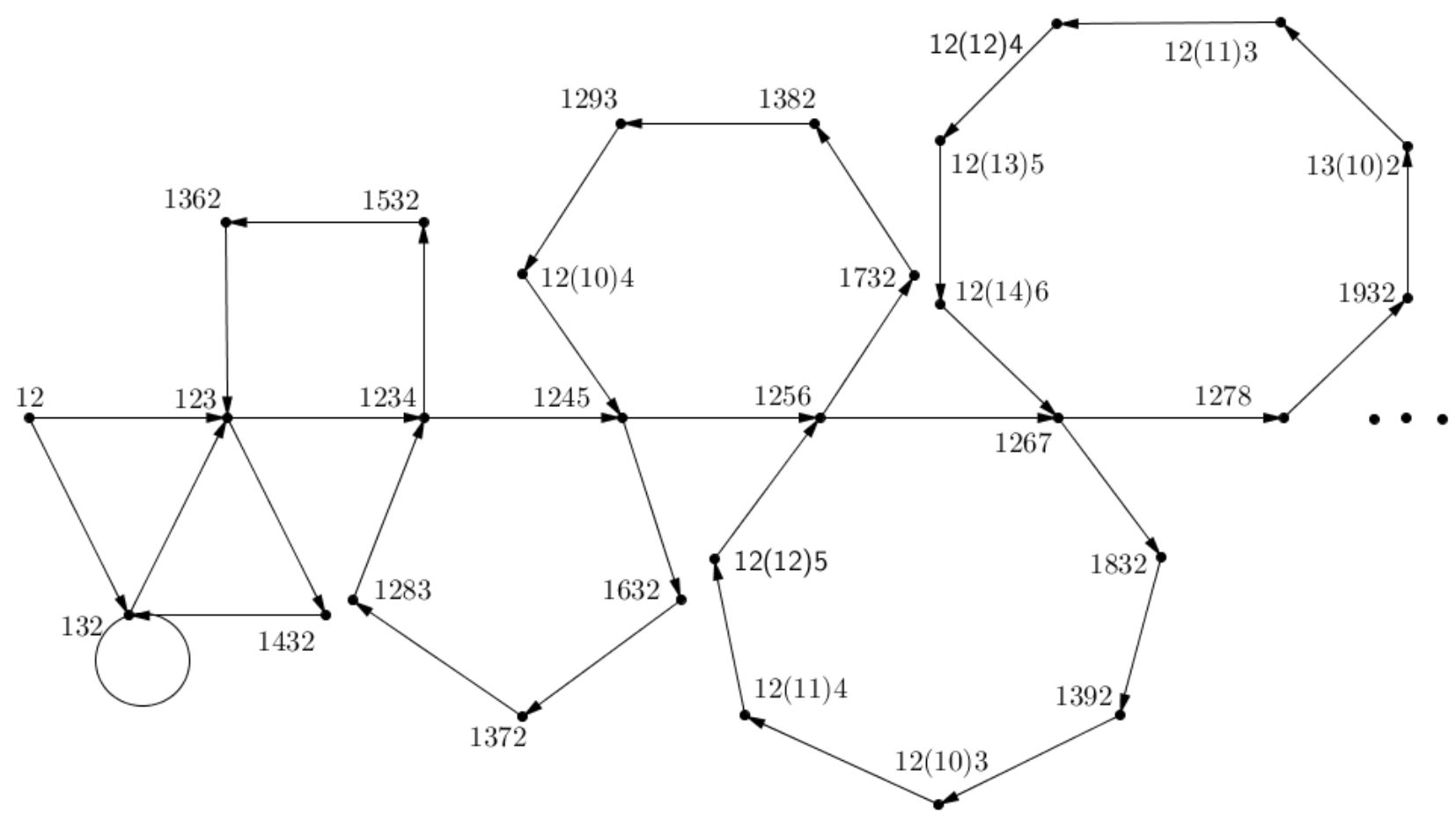

asymptotic lower bound:

$$
f_{1}(n) \geqslant f_{1,-}(n)=\Theta\left(\frac{1}{r}^{n}\right)=\Theta\left(1.5349224995 \ldots{ }^{n}\right)
$$

To find an upper bound, consider the edge from 1267 to 1278 . Because clearly 1267 has more descendants than 1278, we can truncate the graph at 1267 and insert a loop from 1267 to itself. The adjacency matrix for this upper bound graph is the same as the one used above, except that we must insert a 1 into the $22^{\text {nd }}$ row and column. Again, we obtain a rational generating function:

$$
F_{1,+}(x)=\frac{-1-x^{2}+x^{6}+2 x^{7}+x^{8}+2 x^{9}+x^{10}+x^{11}-x^{14}}{-1+2 x-x^{2}+x^{3}-x^{5}-x^{8}-x^{10}+x^{11}-x^{12}+x^{13}+x^{15}}
$$

The denominator of $F_{1,+}$ has a unique minimal root $s=0.65145978572056851317 \ldots$. This gives the asymptotic upper bound:

$$
f_{1}(n) \leqslant f_{1,+}(n)=\Theta\left(\frac{1}{s}^{n}\right)=\Theta\left(1.535014167 \ldots{ }^{n}\right)
$$

We can use the same procedure to estimate the asymptotic growth of $f_{2}(n)$. The digraph used in this case is slightly different:

Similarly, here we will find a lower bound on the growth rate by truncating the graph at the edge between 1278 and 1289, and find an upper bound by creating a loop from 


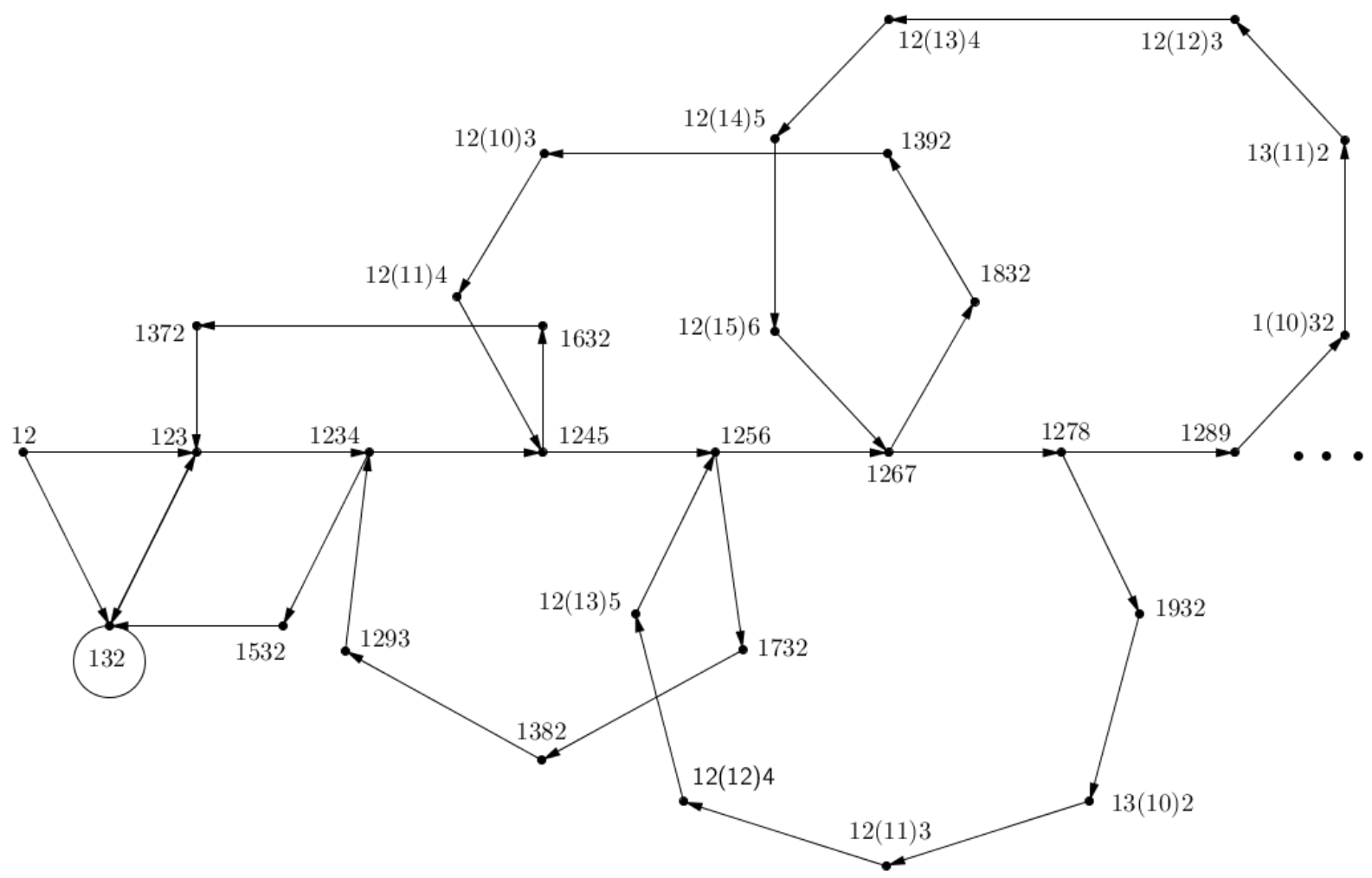

2-convex identically-descending transition digraph

1278 to itself, using the resulting matrices to find generating functions for the cells in the first row:

$$
F_{2,-}(x)=\frac{1+x+x^{2}+2 x^{3}+2 x^{4}+2 x^{5}+2 x^{6}+x^{7}-x^{10}-x^{11}-x^{12}}{1-x-x^{2}-x^{4}-x^{5}+x^{8}+2 x^{9}+x^{10}+x^{11}+x^{12}+x^{13}-x^{14}}
$$

The denominator of $F_{2,-}$ has a unique minimal root $t=0.55979335021175578170 \ldots$, which gives the asymptotic upper bound:

$$
f_{2}(n) \geqslant f_{2,-}(n)=\Theta\left(\frac{1}{t}^{n}\right)=\Theta\left(1.786373489 \ldots{ }^{n}\right)
$$

And,

$$
F_{2,+}(x)=\frac{1+x^{3}-x^{7}-x^{8}-x^{9}-2 x^{10}-x^{11}-x^{12}}{1-2 x+x^{3}-x^{4}+x^{6}+x^{8}+x^{11}+x^{13}-x^{14}}
$$

The denominator $F_{2,+}$ has a unique minimal root $u=0.55977426822528580510 \ldots$, which gives the asymptotic upper bound:

$$
f_{2}(n) \leqslant f_{2,+}(n)=\Theta\left(\frac{1}{u}^{n}\right)=\Theta\left(1.786434384 \ldots{ }^{n}\right)
$$


It certainly appears that $f_{1}(n)$ and $f_{2}(n)$ grow by some asymptotic ratios $C_{1}$ and $C_{2}$, which are around 1.535 and 1.786 , respectively. Theoretically, taking larger and larger transfer matrices will provide us with better and better approximations of these values. It turns out that this is the case, formalized as follows:

Corollary 3.12. For $k=1$ and $k=2, f_{k}(n)$ is $\Theta\left(C_{k}^{n}\right)$ for some finite $C_{k}$.

Proof. We proceed by using subadditivity, as done in [3]. We have that for $k=1,2, f_{k}(m+$ $n) \leqslant f_{k}(m) f_{k}(n)$ since a single-peaked $k$-convex permutation $\pi$ on $[m+n]$ may be uniquely separated out into single-peaked k-convex permutations on $[m]$ and $[n]$. This may be done by first taking the subsequence of $\pi$ consisting of the numbers $[m]$. The other permutation is the permutation on $[n]$ which is order isomorphic to the remaining elements of $\pi$. We have that $\log f_{k}(n)$ is subadditive so by Fekete's lemma we have that

$$
\lim \frac{\log f_{k}(n)}{n}=L .
$$

Given our previous bounds on $f_{k}(n)$, this gives that the limit is finite hence $f_{k}(n)=$ $\Theta\left(C_{k}^{n}\right)$.

Knowing that the constants $C_{1}$ and $C_{2}$ exist, we can obtain better approximations of these values. By considering larger subgraphs than those displayed and repeating the above procedure with (roughly) 500 by 500 transfer matrices, we obtain lower and upper bounds on $C_{1}$ and $C_{2}$ that agree to over 50 decimal places thereby obtaining the values:

$$
\begin{aligned}
& C_{1} \approx 1.53492400555111005892613560688125927773918255689386 \ldots \\
& C_{2} \approx 1.78637686391327203788372322127803476613381673787517 \ldots
\end{aligned}
$$

For any $k \geqslant 3$, we see that $\pi$ is $k$-convex only if it avoids, consecutively, a set $A_{k}$ of permutations. Determining the minimum cardinality of $A_{k}$ is, in general, an open problem. Already, we have seen that $\left|A_{1}\right|=\left|A_{2}\right|=2$. It would be interesting to determine whether our local $k$-convexity condition is strong enough to allow for us to make the following Stanley-Wilf conjecture type claim:

Conjecture 3.13. For each $k>2, \lim _{n \rightarrow \infty}\left(f_{k}(n)\right)^{\frac{1}{n}}=C_{k}$ where each $C_{k}$ is a constant.

As noted by an anonymous referee, the application of the Marcus-Tardos theorem [9] to obtain such a result is tempting. However, $k$-convex permutations are not a class in the strict sense. For instance, consider the 1-convex permutation 46785321. In the permutation containment order, this permutation contains 4567321, which is not 1-convex. Hence as these permutations do not form a class we cannot directly apply the Marcus-Tardos theorem.

\section{A Generating Function for $k=1$}

In this section, we consider the generating function

$$
F_{1}(q):=\sum_{n=0}^{\infty} f_{1}(n) q^{n} .
$$




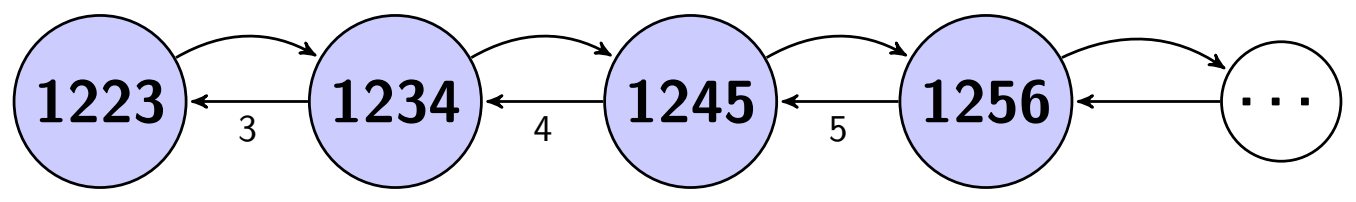

Figure 1: An illustration of the root nodes of $T$.

Note that while we can show that the limit for the growth constant exists and we can produce arbitrarily tight bounds for the limit by using larger matrices, we are still unable to enumerate these permutations exactly. We will simplify a connected piece of the transition digraph, find sufficient information about that piece in order to analyze it, and then use that information in the transition matrix to finish our analysis.

From the 1-convex transition digraph, we see that the nodes that are descended from successive left descendants of 123 are essentially isolated along a path in the graph. We refer to the node 123 as 1223 throughout this section as it simplifies our notation.

To see this, consider a permutation $\sigma$ of the form $12(l)(l+1)$, where $l \geqslant 3$. Note that if this permutation right descends, then the resulting permutation, $R(\sigma)=23(l+2) 1$, will not right descend for $l-1$ generations, and after successive left descents we have $L^{l-1} R(\sigma)=12(l+2+(l-1)) l$. This permutation left descends and right descends and thus by Theorem 3.11 is identically-descending with $12(l-1) l$. Note that as $l \geqslant 3$, the first two entries of the successive left descents of $R(\sigma)$ eventually take the form 12 .

Using this information, we consider the vertex induced subgraph, $T$, induced in the transfer digraph for $k=1$ by the nodes $\{12(k)(k+1): k \geqslant 2\}$. We adopt the convention that an edge labeled with a natural number, $k$, represents a path on $k$ edges from the tail to head of the labeled edge. By the above observations, we see that $T$ has the form illustrated in the figure.

We now wish to count walks in $T$ starting from the node 1234 and ending at 1234 .

Proposition 4.1. Let bot $(q)$ be the generating function counting the number of walks in T from 1223 to 1223 by length. Then,

$$
\operatorname{bot}(q)=\frac{1}{1-\frac{q^{4}}{1-\frac{q^{5}}{1-\frac{q^{6}}{\ddots}}}}
$$

Proof. Let $T_{k}$ be the vertex induced subgraph of $G$ induced by the vertices $\{12(l+1)(l+$ $2): l \geqslant k\}$. Now let $H_{k}(q)$ be the generating function for the number of walks from $12(k+2)(k+3)$ to itself counted by length. So, we see that $H_{1}(q)=\operatorname{bot}(q)$.

Now, note that any walk from 1223 to itself is either the empty walk, or its first step is from 1223 to 1234 and its last three steps return the walk from 1234 to 1223 . Between those steps, the walk consists of a walk beginning at 1234 and returning to 1234, we know these walks are counted by $H_{2}(q)$. So, from the above considerations we have:

$$
H_{1}(q)=1+q^{4} H_{1}(q) H_{2}(q) .
$$


Similarly, by starting our walk in $T_{k}$ and performing the same argument with slight adjustment for the changed path length we see that

$$
H_{k}(q)=1+q^{3+k} H_{k}(q) H_{k+1}(q)
$$

Then by repeatedly substituting the values we found in 4.2 , we are able to solve for 4.1 .

The approach used here to find the generating function is similar to that introduced by Flajolet in [5] as we can associate each walk in our graph with a word in the alphabet $a_{k}, b_{k}$ described in the paper and then evaluate $a_{k}$ as $q$ and $b_{k}$ as $q^{(k+2)}$. More combinatorially, the Motzkin paths corresponding to continued fractions in Flajolet's work correspond to walks in digraphs in our work, where we weight the edges of our walks analogously with the weights given to the edges of the Motzkin paths in Flajolet's work. Further, note that this generating function is quite similar to the generating function for the number of fountains, which has been studied in [6] and [10].

We also wish to find the generating function for the number of walks in $T$ beginning at 1223 counted by length, tot $(q)$, as we will need this later.

\section{Lemma 4.2.}

$$
\operatorname{tot}(q)=H_{1}(q)+q H_{1}(q) H_{2}(q)\left(1+q+q^{2}\right)+q^{2} H_{1}(q) H_{2}(q) H_{3}(q)\left(1+q+q^{2}+q^{3}\right)+\ldots
$$

or

$$
\operatorname{tot}(q)=\sum_{n \geqslant 0} q^{n} \frac{1-q^{n+1}}{1-q} \prod_{i=1}^{n+1} H_{i}(q) .
$$

Proof. The proof of the above is similar to that of Proposition 4.1. Consider the number of walks that end at the first vertex, 1223 ; there are $H_{1}(q)$ of these. Now consider walks ending at 1234; each of these is an extension of a walk ending at 1223, and it takes 1 step to reach this vertex, so there are $q H_{1}(q) H_{2}(q)$ such walks. Next, we note that the number of walks ending at each point along the path back to 1223 from 1234 is then $q H_{1}(q) H_{2}(q)\left(1+q+q^{2}\right)$. We can repeat these observations for each node in the graph and thus arrive at the generating function above.

We now will use the transfer matrix method to find the generating function. To do so, we will integrate what we have learned about $T$ into the transfer matrix framework. Note the following: whenever we consider a walk in the graph that reaches the node 1223, the walk will then branch into other, longer walks staying in $T$ or returning to 1223 before re-entering the bottom of the graph. By "bottom" we mean all vertices not descended from a left descendant of 1223, or in other words, the vertices not in $T$. Instead of keeping track of walks in the upper part of the graph individually, we can use a generating function. To do this consider a walk of length $n$ that ends on 1223; this corresponds to a permutation of length $n+1$. We now wish to determine the number of successive descendants of this permutation, or equivalently the number of walks in the graph beginning this way. The walk will either leave 1223 immediately or continue into $T$. If the walk continues into $T$, then we must keep track of how many walks exist in $T$ after $k$ steps. If it were impossible to leave $T$, we would be done and could simply keep 
track of how many walks there are in $T$ starting at 1223 . However, a walk in $T$ may return to 1223 after some amount of time, at which point the walk branches and leaves $T$, as well as continuing back up into $T$. If we know how many walks of $l$ steps in $T$ begin and end at 1223, we can then anticipate these walks leaving $T$ after a certain number of steps. We already have generating functions for both of these quantities, $\operatorname{tot}(q)$ and $\operatorname{bot}(q) ; \operatorname{tot}(q)$ counts how many walks of length $n$ stay in $T$ if one walk ends at 1223 , and $\operatorname{bot}(q)$ counts how many walks in $T$ beginning at 1223 return to 1223 after so many steps. Thus we see that $\operatorname{tot}(q)-\operatorname{bot}(q)$ has for its $n$th coefficient the number of walks in $T$ of length $n$ beginning at 1223 but not ending at 1223 .

To incorporate this into our transfer matrix framework, we must count both the number of walks in $T$ that will begin at 1223 after so many steps in addition to the number of walks that do not begin at 1223 but remain entirely in $T$. For the number of walks that do begin at 1223 , we can use the generating function $\operatorname{bot}(q)$, as this counts the number of walks that begin and end at 1234 and are entirely in $T$. As bot $(q)$ counts walks by length, we weight the edge between 1223 and 1432 by bot $(q)$ instead of 1 in our transfer matrix. Each time a walk reaches 1223, we will anticipate the future walks that begin at 1223 , but because those walks are $n$ steps in the future, they carry a coefficient of $q^{n}$. Similarly, we wish to count those walks that stay entirely in $T$. We noted before that $\operatorname{tot}(q)-\operatorname{bot}(q)$ counts these walks, and since they have been counted previously these permutations will not have any descendants in our transition digraph. Instead of having an edge from 1223 to 1234 in our transition digraph, we will have an edge to a new node $\operatorname{sink}$, which has no outgoing edges. We will weight the edge to $\operatorname{sink}$ by $\operatorname{tot}(q)-\operatorname{bot}(q)$ and thus have $\operatorname{tot}(q)-\operatorname{bot}(q)$ as the corresponding entry in our transfer matrix. Whenever a walk reaches 1223, exactly two edges will be traversed. One edge returns into the digraph with a weighting reflecting future walks that would be coming from $T$. The other edge goes to a sink and thus records the number of walks that stay entirely within $T$. Now when we consider $\sum A q^{n}$ all of the $q$ coefficients of $\operatorname{tot}(q)$ and $\operatorname{bot}(q)$ simply tell us how many steps in the future a particular walk would take from $T$, even if we did not actually have the steps for that particular walk in the graph.

With the above observations, we can use the following transition matrix to keep track of the number of walks:

$$
M_{1}=\left[\begin{array}{ccccc}
0 & 0 & 0 & 0 & 0 \\
1 & 1 & 0 & 0 & 1 \\
\operatorname{top}(q)-\operatorname{bot}(q) & \operatorname{top}(q)-\operatorname{bot}(q) & 0 & 0 & 0 \\
\operatorname{bot}(q) & \operatorname{bot}(q) & 0 & 0 & 0 \\
0 & 0 & 0 & 1 & 0
\end{array}\right]
$$

Then by recalling that $\left(I-M_{1} q\right)^{-1}=\sum_{n \geqslant 0} M^{n} q^{n}$, we can invert this matrix in Mathematica and sum the first column (as done for finding the approximate growth rates earlier) to find the generating function. Similarly, this generating function only counts half of the walks of length 2 or greater, so we double it and multiply by $q^{2}$ to obtain the final generating function:

$$
F_{1}(q)=1+q-2 q^{2} \frac{1+\operatorname{bot}(q) q^{2}+\operatorname{tot}(q) q}{-1+q+\operatorname{bot}(q) q^{3}} .
$$


The expansion of this generating function begins:

$$
F_{1}(q)=1+q+2 q^{2}+4 q^{3}+8 q^{4}+14 q^{5}+24 q^{6}+40 q^{7}+66 q^{8}+106 q^{9}+170 q^{10}+\ldots
$$

\section{Generating Function for $k=2$}

We can repeat the developments of the above section when $k=2$. We can do this with another transfer matrix and an explicit solution for walks in the corresponding infinite subgraph, which in this case consists of the descendants of the left descendants of 1234 in the graph for $k=2$. The subgraph of interest in this case has structure similar to that from the $k=1$ case, except now the edges that right descend return to nodes two levels below rather than one level below. We now need to keep track of the number of walks in the upper graph ending on 1245 as well as those ending on 1256, as each of these leads back into the original graph. Due to the more complicated structure of this graph we are unable to provide a simple generating function for the number of walks it contains, but if we define $\operatorname{tot}^{\prime}(q), \operatorname{bot}_{1}^{\prime}(q)$ and $\operatorname{bot}_{2}^{\prime}(q)$ where $b^{\prime} t_{1}^{\prime}(q)$ counts walks ending on 1245 and $b_{2} t_{2}^{\prime}(q)$ counts walks ending on 1256 in the upper part of the graph, then we see that the generating function is:

$$
1+q-2 q^{2} \frac{1+q+q^{2}+q^{4}\left(1+\operatorname{bot}_{2}^{\prime}(q)\right)+q^{3}(1+\operatorname{tot}(q))}{-1+q+q^{2}+q^{4}-q^{7} b o t_{2}^{\prime}(q)+q^{5}\left(\operatorname{bot}_{1}^{\prime}(q)+\operatorname{bot}_{2}^{\prime}(q)\right)-q^{6}\left(\operatorname{bot}_{1}^{\prime}(q)+\operatorname{bot}_{2}^{\prime}(q)\right)}
$$

To make use of this formula, one may manually compute the values for the generating functions that it contains. The major difficulty in making use of these generating functions is that we do not have a method for systematically computing $H_{k}(q)$. This suggests that the underlying difficulty in the problem lies with these generating functions and moreover that future work which enumerates walks in highly structured digraphs, such as those given above, should inform the present study. Further, note that solving $\operatorname{tot}^{\prime}(q), \operatorname{bot}_{1}^{\prime}(q)$ and $\operatorname{bot}_{2}^{\prime}(q)$ are not part of the framework of [5] as they are not easily encoded as words over a simple alphabet.

\section{Future Work}

There are many questions that remain to be answered about locally convex permutations and words. A natural goal is to find methods of enumerating these permutations for $k \geqslant 3$, which is more difficult as the permutations can no longer be constructed in such a regular way.

Another question is whether there exists an algebraic generating function for 1-convex and 2-convex permutations. For larger $k$ ? A related question concerns the constants $C_{1}$ and $C_{2}$; we do not believe they are algebraic as the Inverse Symbolic Calculator does not recognize them. In particular, we conjecture:

Conjecture 6.1. The generating functions for 1- and 2-convex permutations are not D-finite.

Furthermore, Conjecture 3.13 stands unsolved. 


\section{Acknowledgments}

The research of both authors was supported by NSF Grant 1263009 and conducted during the Summer 2014 REU program at East Tennessee State University. We would like to thank Bill Kay for suggesting the problem and Anant Godbole for his suggestion about using subadditivity.

\section{References}

[1] M. H. Albert, M. Elder, A. Rechnitzer, P. Westcott, and M. Zabrocki. On the Stanley-Wilf limit of 4231-avoiding permutations and a conjecture of Arratia. Advances in Applied Mathematics, 36(2):96-105, 2006. Special Issue on Pattern Avoiding Permutations.

[2] M. H. Albert, S. Linton, N. Ruškuc, V. Vatter, and S. Waton. On convex permutations. Discrete Mathematics, 311(8-9):715-722, 2011.

[3] R. Arratia. On the Stanley-Wilf conjecture for the number of permutations avoiding a given pattern. Electronic Journal of Combinatorics, 6(1):1-4, 1999.

[4] P. Brändén and T. Mansour. Finite automata and pattern avoidance in words. Journal of Combinatorial Theory, Series A, 110(1):127-145, 2005.

[5] P. Flajolet. Combinatorial aspects of continued fractions. Discrete Mathematics, 32(2):125-161, 1980.

[6] M. Glasser, V. Privman, and N. Svrakic. Temperley's triangular lattice compact cluster model: exact solution in terms of the $q$ series. Journal of Physics A: Mathematical and General, 20(18):L1275-L1280, 1987.

[7] S. Kitaev. Patterns in Permutations and Words. Springer, 2011.

[8] T. Mansour and A. Vainshtein. Restricted permutations and Chebyshev polynomials. Séminaire Lotharingien de Combinatoire, 47:Article B47c, 17, 2002.

[9] A. Marcus and G. Tardos. Excluded permutation matrices and the Stanley-Wilf conjecture. Journal of Combinatorial Theory, Series A, 107(1):153-160, 2004.

[10] A. Odlyzko and H. Wilf. The editor's corner: $n$ coins in a fountain. American Mathematical Monthly, pages 840-843, 1988.

[11] R. Stanley. Enumerative Combinatorics, volume 1. Cambridge University Press, 2011.

[12] V. Vatter. Finitely labeled generating trees and restricted permutations. Journal of Symbolic Computation, 41(5):559-572, 2006.

[13] H. Wilf. Generatingfunctionology. Academic Press, 1994. 